\title{
Theoretical Aspects of the Optimization of Parameters and Discretization of Control of Processing and Metallurgical Production's Technology Products
}

\author{
Yuriy Genrikhovich Lobotskiy, Valeriy Vasilyevich Khmara* \\ Industrial Electronics Department, North-Caucasian Mining and Metallurgical Institute (State Technological \\ University), Vladikavkaz, Russian Federation; khmaraval@yandex.ru
}

\begin{abstract}
This article deals with the issues of optimizing the number of technology products controlled in the Automated Systems for Analytical Control (ASAC) and the elements identified in them, as well as optimal control over the discretization of sending composited samples to analysis. The article discusses the developed and practically applied method and algorithm for calculating the optimal time interval between proximate measurements of the same technology product. Scientific novelty of the proposed method and algorithm is in consideration of the real rate of change of the controlled parameter. The article is meant for developers and users of Automated Systems for Analytical Control (ASAC) of the material composition of recycled technology products.
\end{abstract}

Keywords: Analytical Control, Composited Sample, Representative Snap Sample, Sampling Algorithm, Sampling and Reconstruction of Continuous Information, Technological Product Testing

\section{Introduction}

At modern concentrating, metallurgical and many other processing industry companies high technological performance largely depends on the proper organization of quality assurance of processed technology products at each stage of the technological process. As a rule, information on the quality of processed products is obtained by chemical analysis, by which one means a set of actions that are aimed at obtaining information on the chemical composition of the object under research.

Chemical analysis is a complex multi-step process. One can identify the following stages in any object analysis: Task-setting, choosing the method and analysis layout, selecting a representative sample of the controlled product, preparing the sample for analysis, measurements, processing of measurement results. This division is conventional: Each step may be relatively complex and consist of many individual steps.
The most important stage of chemical analysis is the sampling of a representative sample, as errors in the sampling cannot be corrected by any analytical or mathematical methods. Practical application of the analysis is based on the internal belief that the analysis results obtained for this sample are also applicable to the entire mass of the material it is taken from. This is true only provided the chemical composition of the sample correctly reflects the composition of the entire mass of the material. The term "sampling" refers to operations that determine methods of sampling a sufficient quantity of the material, which would represent the entire tested product. Frequently, sampling is the most difficult stage of analysis, especially when analyzing industrial raw materials, supplied by lots of several thousand tons. Since the value of a lot is determined by the mass of individual components of raw materials, rather than its total mass, chemical analysis should establish the ration between these values.

\footnotetext{
*Author for correspondence
} 
The weight of the sample at the final stage of the sampling process can vary from a few grams to several hundred grams. And though it can be only one of the fifty-millionth part of the total mass of the lot, the sample composition should be as close to the average composition of the total mass as possible. It is clear that the reliability of the analysis cannot exceed the reliability of sampling: even the most careful analysis of a badly taken sample is just a waste of effort.

Methods for sampling and its size are determined, first of all, by the physical and chemical properties of the object being analyzed. It is necessary to take the following into account while sampling:

- Physical state of the analyzed product;

- Heterogeneity of the analyzed material and the size of solid parts the heterogeneity starts with;

- The required accuracy of the estimation of the components' content throughout the mass of the analyzed product.

This article presents the pressing issues of optimizing the number of controlled technology products and the elements identified in them, as well as the optimal control of the discretization of automatic instrumental analytical control of composited samples of each controlled technology product that depend directly on the response time of the controlled technological process. The purpose of this article is to discuss the developed and practically applied method and algorithm for calculating the optimal time interval between successive measurements of the same technology product, which take into account the actual speed the controlled parameter changes with. The findings described herein have been used in the operating automated systems for analytical control of concentrating and metallurgical plants and can be recommended to be used in the newly created ones. The article is meant for those who are focused on the creation or operation of Automated Systems for Analytical Control (ASAC) of the material composition of recycled technology products.

\section{Literature Review}

The necessary analytical information about the quality of the original raw materials, middling products, finished goods and dump waste products at concentration and metallurgical plants is obtained by testing, that is, by taking samples and subsequently analyzing representative samples that reliably characterize the entire mass of the original flow of controlled product ${ }^{1}$. In production conditions, while organizing continuous monitoring of the quality of processed raw materials, middling products, finished products and tailings, one takes considerable efforts to ensure the required discretization of primary snap samples of each tested technology product. This requirement is regulated and ensured by the necessary ratio between a snap sample scope and the scope of the tested technological flow, taking into account the maximum size of the solid phase in tested product ${ }^{2}$. A composited sample of the technological process is made from the taken snap samples during a certain time period, and such sample in its composition must be identical to the entire sampled lot of the controlled product. The probability of compiling quite representative composited samples with any set accuracy is determined by a number of individual lsnap samples that make up the composited one. The compiled composited sample is reduced to the required amount and is sent to express laboratory for analysis. As the requirements to the accuracy and speed of obtaining the analytical information on the composition of processed products are rather high, it is usually prepared in the Automated Systems of Analytical Control (ASAC) $)^{3,4}$.

E.L. Itskovich ${ }^{5}$ was the first to consider the issues of determining the required frequency of measurements during discontinuous control in 1961. The work of A.S. Kasatkin ${ }^{6}$ is dedicated to methods of finding the minimum set of monitored parameters that provide for the desired probability of facility operations.

Then, once the industry started using computers, E.L. Itskovich $^{7}$ revisited the issues of identifying the required frequency of measurements during discrete control, but using modern - at the time - computers to calculate the discretization and process the analytical information. V.V. Pismenny devoted his research to the same issues ${ }^{8}$.

In 1975, R.L. Stratonovich ${ }^{9}$ introduced the concept of the value of information that is characterized by the maximum benefit, which this information can give for reducing losses. The benefit of the information is that it reduces the losses associated with average penalties. At that, a function is set that assigns smaller penalties or greater rewards for more successful action than for less successful one. The goal is to minimize the average penalties. However, it is difficult to apply the concept to the information value to measurement data, since in this case loss assessment does not take account the characteristics of the information receiver and, moreover, one often has to intuitively choose the loss function.

At about the same time, when creating first Automated Systems of Analytical Control (ASAC) and selecting 
the list of controlled products and components, an information criterion proposed by E. Ya. Ovcharenko ${ }^{10}$ was often used.

By 1980, commercial operation of the first ASAC started at the largest metallurgical plants, and therefore the issues of information exchange between ASAC and Automatic Process Control Systems (APCS) become highly relevant ${ }^{11}$.

A little later serious works appeared concerning the issues of evaluating the economic efficiency of applying the automated systems and of analytical control devices in non-ferrous metallurgy ${ }^{12}$.

An effective mathematical tool for creating a mathematical model of the control discretization is the artificial neural networks method ${ }^{13}$.

The Eurasian National University named after Gumilev in Astana examined the issues of uniform discretization with the frequency criterion quite deeply ${ }^{14}$.

At the current stage, the main requirement for ASAC is to minimize the control parameters, the range of which reflects the course of the technological process best and is used to manage that technological process that largely determines the effectiveness of the system of analytical control $^{15}$. The issue of control parameters' minimization is particularly relevant at the stage of ASAC creation.

\section{Methods of Calculating the Minimal Number of the Controlled Parameters and Elements}

If the analytical information obtained in ASAC is used in the mathematical model of the Automatic Process Control System (APCS), the list of controlled parameters is determined by the key tasks dealt with by APCS. The most general approach to the selection of controlled parameters means that data losses associated with the lack of control of this or that parameter are analyzed. A list of the controlled parameters is made so that the information loss after control does not exceed a set level ${ }^{10}$.

To simplify the methodology for determining the optimal number of monitored parameters one can conventionally accept the control system as ideal, choose list of controlled parameters by the close criterion of the control process effectiveness, and then refine it based on their efficiency criterion, taking into account the actual characteristics of the control and management system.
If the significance of losses on each output parameter of the object is equal, it is possible to find a minimum set of controllable parameters that ensure the set probability of the object functioning ${ }^{6}$.

Let the controlled object be characterized with parameters $\mathbf{a}_{1}, \mathbf{a}_{2}, \ldots, \mathbf{a}_{\mathbf{n}}$ that determine its operating capacity. Let us mark the event of the operating capacity per parameter $\mathbf{a}_{i}$ viaA $\mathbf{A}_{i}$. As the operating capacity of the object is determined by the operating capacity per each parameter, the probability of the operating capacity of the object - subject to the ideal control system - equals the probability of the fail-safe performance of the object for all parameters:

$P=P\left(A_{1}, A_{2}, \ldots, A_{n}\right)=P\left(A_{1}\right) \cdot P\left(A_{2} / A_{1}\right) \cdot P\left(A_{3} / A_{1} A_{2}\right) \cdot \ldots$

$\cdot \mathrm{P}\left(\mathrm{A}_{n} / \mathrm{A}_{1} \mathrm{~A}_{2} \ldots \mathrm{A}_{n-1}\right)=\prod_{i=1}^{n} \mathrm{P}_{i / i-1}$

where: $P\left(A_{i} / A_{1} A_{2} \ldots A_{n-1}\right)=P_{i / i-1}$-the probability of the failfree operation of the object for parametera ${ }_{i}$, calculated subject to the condition that the subject is operational from $\mathrm{a}_{1}$ to $\mathrm{a}_{\mathrm{i}-1}$.

By the fail-free operation of the parameter we mean the consolidated indicator that includes operating capacity of the standards, methods of control, analysis itself, validation of the analysis findings, provision of the ready analytical information to consumers, etc.

Depending on the order of selecting parameters a one can get $\mathbf{n}$ ! combinations of products that produce one and the same numerical value P. To minimize the number of the controlled parameters it is advisable for the purpose of control to first select the parameter with the lowest probability of fail-free operation. For this purpose, probabilities of the fail-free operation are calculated for each parameter and the least reliable parameter $\mathbf{a}_{j}$ is selected. Then one can calculate the probability of the fail-free operation for other parameters provided that parameter $\mathbf{a}_{j}$ is operational, and once again select the least reliable parameter. Such sequence of operations is conducted until the following ratio is met:

$\mathrm{P}_{\Pi} \leq \prod_{i=1}^{n_{i}} \mathrm{P}_{k_{i / i-1}}(\tau) \prod_{j=n_{i}+1}^{n} \mathrm{P}_{n_{j / j-1}}(t)$

where: $\mathrm{P}_{n_{j / j-1}}(t)$ - is the conditional probability of the fail-free operation under the $\mathrm{j}$ non-controlled parameter during the time interval $t$;

$\mathrm{P}_{k_{i l i-1}}(\tau)$ - is the conditional probability of the fail-free operation under the i controlled parameter by the time $\tau$ after the control;

$n_{i}$ - the number of controlled parameters. 
The controlled parameters are minimized based on the information criterion. For a multi-element analytical control the product entropy is determined according to the formula:

$H_{\mathrm{Pr} .}(t, \tau)=\int f(t, \tau, \chi) \log _{2} f(t, \tau, \chi) d x$

where: $f(t, \tau, x)$ - is the multidimensional differential law of the control parameters' probability distribution.

The entropy of each product should be determined by the formula:

$H_{\mathrm{Pr} .}(t, \tau, \chi)=\log _{2} \sigma_{\chi} \sqrt{2 \pi e}$

The entropy of independent controlled elements in the product should be determined by the formula:

$H_{\text {El.pr. }}(t, \tau)=\sum_{i=1}^{n} H\left(t, \tau, \chi_{i}\right)$

where: $i$ - is the number of controlled elements.

Minimization of the controlled items in this product at a given level of uncertainty is achieved through a consistent removal of the parameters that have the greatest uncertainty, until the following correlation is met:

$H_{E l . p r .}(t, \tau) \geq \sum_{i=1}^{n} H\left(t, \tau_{1}, \chi_{i}\right) \rightarrow \min$.

If the values of the parameters are not the same, and during the system development a higher reliability for the most important parameters was included, it is necessary to ensure the highest probability of fail-free operation under these specific parameters.

Information characteristics that allow analyzing the operations of both the system as a whole and individual automatic and non-automatic devices have the highest integral criterion.

In reality the requirements to the system of analytical control are most often designed in cooperation with process engineers and designers of APCS, if it is planned to make a technological process automated. The Delphi approach can also be used for this purpose, where process engineers and leading operators of production act as experts.

The list of monitored parameters may include such parameters which allow - during the introduction of the system - to study the process in more detail and thereby identify more effective ways and methods of control. While operating the system of analytical control it might become unnecessary to control some parameters, but there will be new ones, for which the system should be improved. This process is completely natural and can be repeated many times during the operation of ASAC.

To determine the optimal number of monitored parameters the following sequence can be recommended:

- If you have a mathematical model, developed as the result of technological process examination and used in the APCS for the purpose of optimization, the analytical parameters that are part of the mathematical model should be controlled. The very fact of an analytical parameter presence of a technological process indicates its importance and the need for control.

- In the absence of previously developed requirements, the latter are formed together with process engineers and the personnel engaged in the direct control of technological processes. Clearly, with the increasing number of monitored parameters (complete nature of control) the accuracy of control increases. However, too high control accuracy and an unnecessarily large number of controlled parameters lead to an unjustified increase in the time spent on the object verification, more complex ASAC, growth of its cost and reduced reliability ${ }^{12}$. Therefore, it is advisable to minimize the controlled parameters using the information criterion as sufficiently effective for this purpose.

\section{Development of Principles for Optimizing the Discretization of Analytical Control}

The efficiency of automated systems of analytical control can be significantly reduced if the processed result of a technology product analysis arrives to the consumer with a considerable time delay or does not reflect the objective changes in the quality of the product during the technological process.

Technological processes of non-ferrous metallurgical industry, as a rule, are very inert that enables a discrete analytical control of the composition of processed products. No less important factor is the lack of technical means of analytical control that have the necessary characteristics during the continuous analysis of the majority of products directly in the technological stream. That is why in most cases such analytical devices are used in ASAC that envisage discrete, sequential, multi-element analysis of the samples prepared. Such devices, for example, include the $\mathrm{X}$-ray spectral and atomic absorption 
analyzers, optical spectrometers, etc. In this regard it is necessary to set such a discretization for sending representative samples for analysis that will help manage the process optimally. Optimal is a discretization, which will provide for a representation of analytical information to the original function with a given accuracy and with a minimum number of samples. In this case, all the analysis results are essential for the original function restoration.

The main indications of the difference in the methods of discretization and reconstruction of continuous functions are ${ }^{5}$ :

- The regularity of samples;

- Samples' selection criteria and evaluation of fidelity;

- Reproduction method;

- Type of the reproducing function.

Regularity of samples envisages two main groups: A uniform and non-uniform discretization, i.e. adaptive discretization, which in turn can be a multiple or non multiple intervals.

The following can be used as a criterion of the discretization for the continuous process control:

- Kotel'nikov's frequency criterion ${ }^{16}$, in which the intervals between samples are selected based on the frequency spectrum of the sampled signal;

- Correlation criterion of samples;

- Quantum criterion of samples.

Below we consider the criteria of the reproduced function $y(t)$ deviation from the original signal $x(t)$ at each discretization intervals $\tau_{i}$ :

The largest deviation criterion:

$\varepsilon_{j(t)}=\sup \left|\chi_{y}(t)-y_{y}(t)\right|$

Root mean square criterion:

$$
\varepsilon_{y}^{-2}(t)=\frac{1}{\tau_{j}} \cdot \int_{t o(j-1)}^{t_{o j}}\left[\chi_{j}(t)-y_{j}(t)\right]^{2} d t
$$

where: $t \in\left[t_{o(j-1)}, t_{o j}\right]$

Integral criterion:

$\overline{\varepsilon_{j}}(t)=\int_{t_{o(j-1)}}^{t_{o j}}\left|\chi_{i}(t)-y_{i}(t)\right| d t$

where: $t \in\left|t_{o(j-1)}, t_{o j}\right|$;

probability-zone criterion:

$P\left[\chi_{j}(t) \in S_{i}\right]=1-P\left[\chi_{i}(t) \in \overline{S_{i}}\right]$ where: $P\left[\chi_{i}(t) \in \bar{S}_{i}\right]$ is the probability of value $x(t)$ exiting

the zone $S_{i}$ at interval $\tau_{i}$.

Reproduction of the original signal can be done in two ways: By extrapolation and interpolation.

The methods of discretization and extrapolation of reproducing functions do not require signals' delay within the range of discreteness. Consequently, they can be used in the systems that operate in real time (in control system). To analyze the dynamic characteristics of the controlled variable in order to study the technological process, adjust the interval between samples at a uniform discretization and calculate the technical and economic indicators the interpolation methods are used.

Kotelnikov's theorem is of limited use, as it is accurate for functions with a limited range that belong to a degenerated class. For these functions, using their previous values and through the linear forecast one can be predict its future values in any subsequent time with the mean square error, as little different from zero as desired. Presentation of a continuous function in the form of discrete samples at a time interval $\Delta_{\mathrm{T}}=\frac{1}{2 \mathrm{fm}}$ does not allow for an accurate reproduction of the process developing in time.

Two discretization principles are of interest: The first one allows to restore accidental stationary signals by the linear forecast system with the mean root square error $\bar{\sigma}^{2}$ , as little different from zero as desired, within the time interval equal to the correlation interval $\tau^{0}$. It is known that for an uninterrupted signal of final duration $\mathrm{T}$ the number of correlated samples equals:

$\mathrm{N}=\frac{T}{\tau_{\mathrm{o}}}$

Then the correlation interval is determined with the help of efficient frequency band for occasional nonstationary signals according to the formula:

$\tau_{o}=\frac{1}{2 \Delta F_{E F F}(t)}$,

where: $\Delta F_{E F F}(t)$ - is the effective frequency band of instantaneous spectral density up to a constant factor.

In determining the reference period for the uniform sampling under the second method the fidelity is estimated by a mean-root-square deviation at the nodal points:

$\Delta_{T}=B_{\chi \chi}(0)-\frac{\overline{\varepsilon^{2}}}{2}$, 
where: $B_{\chi \chi}^{-1}$ - is the function contrary to the correlation one.

The experimental data $(30-50$ value measurements with interval $\left(h_{\beta}\right)$ is used to build a diagram $\sigma^{*}=f\left(h_{\beta}\right)$, which is an approximate estimate of the mean root square deviations of the value during the time intervals divisible by $h_{b}$. Setting the permissible $B_{\chi \chi}^{-1}$, one determines the sampling time $\Delta \mathrm{T}$.

Comparison of the errors when using different formulas for extrapolation and interpolation ${ }^{7}$ demonstrates that using a large number of members in a statistical interpolation polynomial with the adopted approximation of the correlation function is inadvisable, since in this case the error is reduced slightly. In all cases statistical interpolation is preferable to the parabolic one, since in this case error is several percent lower on average.

\section{Uniform Sampling with Multiple Intervals}

While creating ASAC it is sufficient to define the discreteness of control using experimental data, which at relatively low cost makes a fairly good approximation of the real process ${ }^{10}$. While implementing and operating the system it is absolutely necessary to correct the sample period, which is conditioned by the natural changes in the statistical characteristics of the processed feed materials.

Such adaptive algorithms require statistical processing of the current measurements' results. The sliding implementation of measurement determines the current value of the correlation function, on the basis of which the sampling frequency varies. The disadvantage of this method is the need to memorize a sufficiently large amount of data that requires a significant amount of memory and a large number of elementary arithmetic operations. To eliminate this drawback we propose a method which is suitable for both stationary and nonstationary processes ${ }^{11,15}$. Based on this method, an algorithm was designed that adjusts the time interval between measurements depending on the specific implementation of the process within a reasonable time interval, a block-diagram is shown in Figure 1.

Discretization is performed with a multiple interval

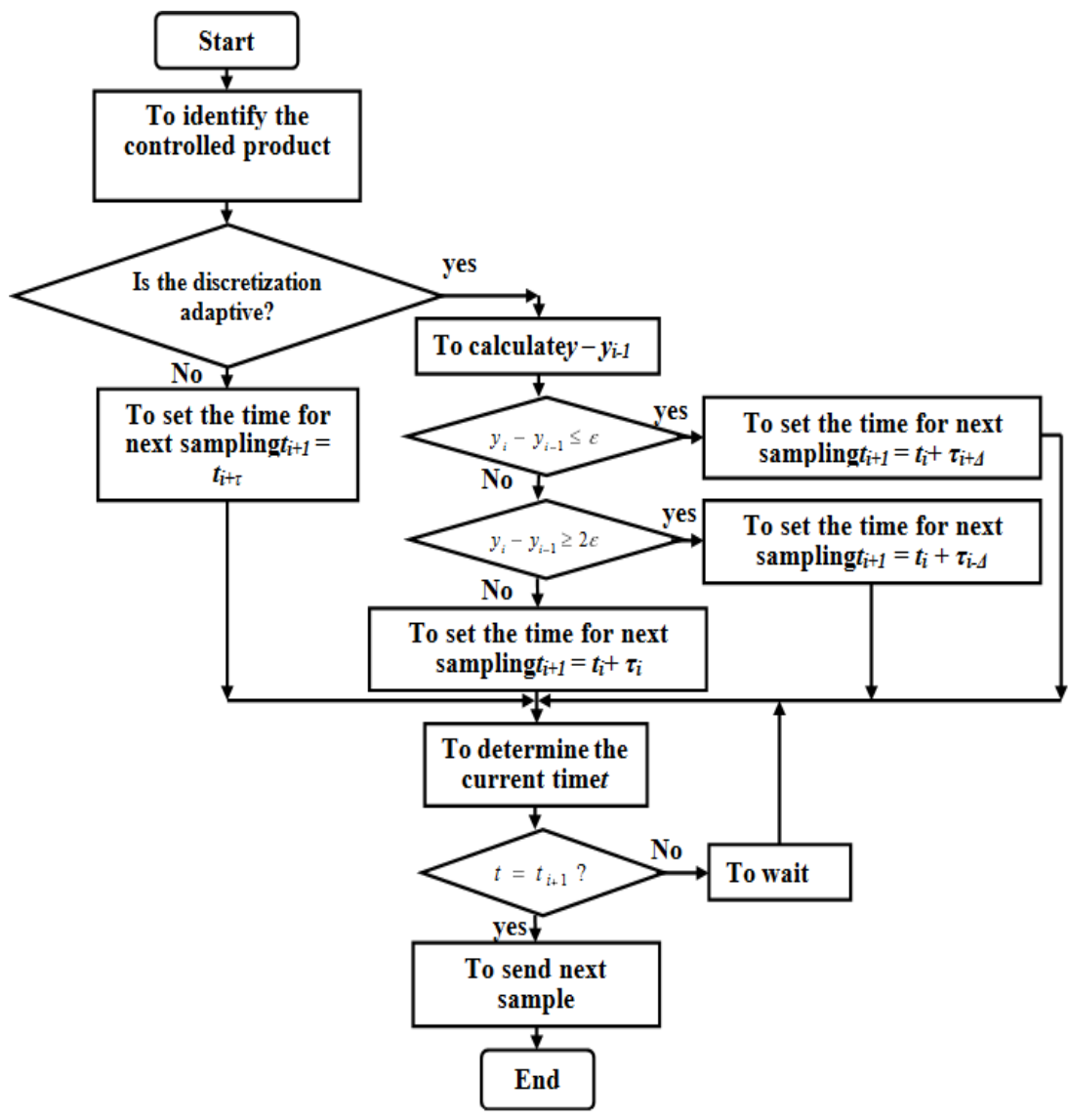

Figure 1. Algorithm of the adaptive uniform sampling of control 
according to the rule:

$\left\{\begin{array}{c}\tau_{i+1}=\tau_{i} \text { if } \varepsilon \leq y_{i}-y_{i-1} \leq 2 \varepsilon \\ \tau_{i+1}=\tau_{i}+\Delta \text { if } y_{i}-y_{i-1} \leq \varepsilon \\ \tau_{i+1}=\tau_{i}-\Delta \text { if } y_{i}-y_{i-1} \square \geq 2 \varepsilon\end{array}\right.$

where: $\tau_{i}, \tau_{i+1}$ is the duration of the time interval between the measurements set at the $i$-th and $(i+1)$ th step of the control;

$\Delta$-is the fixed value by which the duration of the time interval can change at each measurement step;

$y_{i}$ and $y_{i+1}$ - measurement results at the $i$ th and $(i-1) t h$ measurement step;

$2 \varepsilon$-permissible deviation of the adjacent measurement results.

Application of the adaptive algorithm allows reducing the number of measurements by 4 to 6 times compared with the control algorithm at a rigid schedule. This not only reduces the number of analyzers, but also cuts down the cost of analytical control.

The analytical control usually requires a significant investment of time from the time of sampling the controlled product to the time the finished result of the analysis is issued. This time consists of the sum of the periods of time required to perform the operations: Taking of a representative sample, preparation of the taken sample for shipment, sample delivery to the analytical laboratory, preparation of the sample for analysis, the actual analysis and processing of the analysis results $t_{\text {pros }}$.

Time $t_{\text {pros }}$ is taken into account by the additional extrapolation. At that, the actual period of sampling $\left(t_{o}\right)_{a c t}$ is determined according to the formula:

$\left(t_{o}\right)_{\text {act }}=t_{o}-t_{\text {pros }}$,

It is obvious that if $t_{\text {pros }}$ is comparable with the calculated sampling period $t$, the application of the discrete control might become inexpedient. Then it is necessary to use a continuous analyzer or look for ways to reduce the $t_{\text {pros }}$. If there is no acceptable solution, one should decide whether it is expedient to control the parameter.

Let us analyze some options for the material flows' control.

The purpose of the analytical control is to determine the concentration of elements or their compounds in industrial products or finished products for a specified length of time.

However, determining the material composition of the product is only a part of the necessary measurements, which include the measurement of weight, moisture, costs, etc. The correlations detected in time when monitoring a material are conditioned by the spatial distribution of the element in the product. The larger the material consumption, the greater the speed of the controlled components advancing and, accordingly, dynamic changes of the parameter over time. In the stationary distribution of the element in the material the time of the correlation function' decline $\mathrm{K}(\tau)$ depends on its consumption.

To control the solid material flow it is necessary to measure three components for more complete and accurate assessment of the controlled element consumption:

- Product consumption per time unit расход, $Q$ $\mathrm{t} / \mathrm{h}$;

- $\quad$ Product moisture level, W\%;

- Composition, C \%.

Then the controlled element's consumption will be determined according to the formula:

$q_{e l}=\frac{C Q}{100}\left(1-\frac{W}{100}\right)$

After linearization it would be possible to determine the need for the control over $C, Q$ and $\mathrm{W}$ :

$\frac{d q_{e l}}{d Q}=\frac{C}{100}\left(1-\frac{W}{100}\right)$

$\frac{d q_{e l}}{d C}=\frac{Q}{100}\left(1-\frac{W}{100}\right)$;

$\frac{d q_{e l}}{d W}=-\frac{C Q}{10000}$;

$\delta_{\Sigma}^{2}=\delta_{Q}^{2}\left(\frac{d q_{e l}}{d Q}\right)^{2}+\delta_{C}^{2}\left(\frac{d q_{e l}}{d C}\right)^{2}+\delta_{W}^{2}\left(\frac{d q_{e l}}{d W}\right)^{2}$,

where: $\delta_{Q}$ - mean root square deviation of consumption; $\delta_{C}$ - mean root square deviation of concentration;

$\delta_{W}$ - mean root square deviation of moisture content.

With low $\delta_{W}$ moisture content of the product can be

disregarded, as $\frac{C Q}{10000}$ value is sufficiently small.
Then:

$\delta_{\Sigma}^{2}=\delta_{Q}^{2}\left(\frac{d q_{e l}}{d Q}\right)^{2}+\delta_{C}^{2}\left(\frac{d q_{e l}}{d C}\right)^{2}$

$\delta_{\Sigma}^{2}=\delta_{Q}^{2}\left[\frac{C}{100}\left(1-\frac{W}{100}\right)\right]^{2}+\delta_{C}^{2}\left[\frac{Q}{100}\left(1-\frac{W}{100}\right)\right]^{2}$

This formula helps determine the expediency of control over the product concentration and consumption.

Let us look at potential cases:

1. $\delta_{Q}^{2}\left[\frac{C}{100}\left(1-\frac{W}{100}\right)\right]^{2} \approx \delta_{C}^{2}\left[\frac{Q}{100}\left(1-\frac{W}{100}\right)\right]^{2}$, 
i.e. an error entered when determining the consumption can be compared to the error of determining concentration. This means that a comprehensive measurement of concentration and consumption is required.

2. $\delta_{Q}^{2}\left[\frac{C}{100}\left(1-\frac{W}{100}\right)\right]^{2}>>\delta_{C}^{2}\left[\frac{Q}{100}\left(1-\frac{W}{100}\right)\right]^{2}$,

In this case the control of the material consumption should be ensured first of all.

$3 . \delta_{Q}^{2}\left[\frac{C}{100}\left(1-\frac{W}{100}\right)\right]^{2}<<\delta_{C}^{2}\left[\frac{Q}{100}\left(1-\frac{W}{100}\right)\right]^{2}$,

In this case, first of all the element concentration in the material should be controlled.

In the latter two limit cases it's possible to use adaptive sampling method.

In the first case, the adaptation should be carried out on the product flow, as it has the greatest influence on the accuracy of $q_{e l}$ determination, whereas in the second case - on the concentration of the element to be determined. This adaptive control of the consumption is carried out quite easily as it is possible to monitor it continuously.

\section{Variable-Part Sampling with Aliquant Intervals}

However, the adaptive algorithm considered above used for the optimization of the time interval between sending composited samples of one and the same technology product for analysis does not fully take into account the dynamics of the key controlled elements in the analyzed composited samples. The reason is that the above algorithm assumes that the rate of changes of the main monitored parameters during a fairly long period of time (much more than the average duration of compiling a composited sample) remains approximately constant. In real technological process the rate of changes of those main controlled parameters can dramatically change depending on many both technological and original "raw material" factors.

Based on this, we have developed and tested an adaptive algorithm for the optimization of time intervals between successive sending of composited samples of controlled technology products for analysis, taking into account changes in the speed (first derivative values) of the measured technological parameter. This technique helps to more accurately determine the optimal interval between two successive measurements of one and the same technology product.

The computer-based algorithm for calculating and setting the time interval between adjacent sending of doses of averaged samples for instrumental analysis, taking into account the rate of change in the controlled parameter is shown in Figure 2.

In this case, the discretization is carried out according to the rule:

1. When the system of the automatic selection and delivery of samples for analysis starts, we set the frequency of the analysis of the dosed amounts of averaged sample of each technology product, calculated according to the V.A. Kotelnikov's theorem:

$\Delta \mathrm{t}=1 /\left(2 \mathrm{~F}_{\max }\right)$,

where: $\mathrm{F}_{\max }$ is the maximum frequency of changes in the main controlled parameters in the given technology product.

According to the Kotelnikov's theorem, it is not necessary to obtain an infinite aggregate of all values of the measured parameter of the controlled technology product, but rather only those of its values, which are spaced from each other at a distance $\Delta \mathrm{t}=1 /\left(2 \mathrm{~F}_{\max }\right)$.

2. After the system of samples' automatic selection and sending for analysis reaches the steady state of operation, the following rule is used to determine the frequency of sending for analysis an averaged sample of each tested technology product:

$\left\{\begin{array}{l}\tau_{i+1}=\tau_{i}, \text { if } v \leq v_{i}=\frac{y_{i}-y_{i-1}}{t_{i}-t_{i-1}} \leq 2 v, \\ \tau_{i+1}=\tau_{i}+\delta_{i}, \text { if } v_{i}=\frac{y_{i}-y_{i-1}}{t_{i}-t_{i-1}} \leq v, \\ \tau_{i+1}=\tau_{i}-\delta_{i}, \text { if } v_{i}=\frac{y_{i}-y_{i-1}}{t_{i}-t_{i-1}} \geq 2 v,\end{array}\right.$

where: $\tau_{i+1,} \tau_{i}, \tau_{i+1}$ - the duration of time interval between the measurements set at the (i-1)-th, i-th and at $(i+1)$-th control step;

$\mathrm{y}_{i}$ and $\mathrm{y}_{i-1}-$ results of measurements at the $\mathrm{i}$-th and at (i - 1)-th control step;

$\delta_{i}=\tau_{i} \frac{\left(\nu_{i}-\nu_{i-1}\right)}{\nu_{i}}$ - the calculated duration of the time interval by which the length of the previous time interval should be changed when setting the next duration of the 


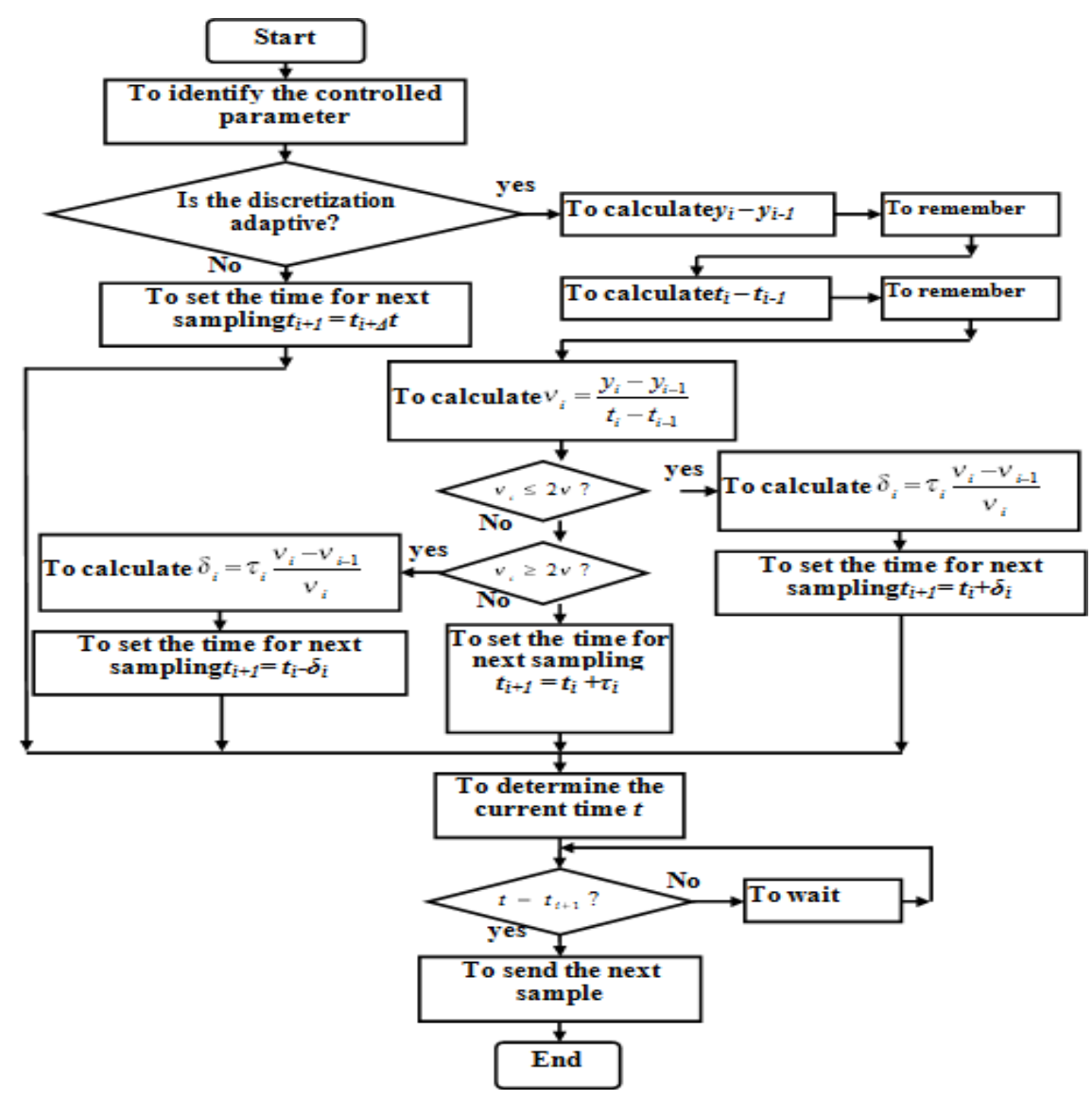

Figure 2. The algorithm of the adaptive non-uniform discretization of control

time interval;

$t_{i}$ and $t_{i-1}$-current time of sending a sample for analysis at the $\mathrm{i}$-th and the (i-1)-th control step;

$2 v$-permissible deviation of the adjacent measurement results.

\section{Conclusion}

The specifics of the proposed adaptive algorithm for the optimization of time intervals between successive sending of composited samples of the controlled technology products for analysis, taking into account the changes in the speed of the measured technological parameter are the following:

- As a predictive model for calculating the next time interval to produce a composited sample of the controlled product, one can use the non-linear system of ordinary differential equations.

- The proposed approach to adaptive discretization of automatic analytical control of technology products of processing and metallurgical industries takes into account the constraints that are imposed on both the controlled variables, and the components of their state vectors.

- The proposed approach to adaptive discretization of the automatic analytical control of technology products of processing and metallurgical industries envisages a minimization of the function that characterizes the quality of the analytical control of technology products in real time.

- To be able to control, including the predictive adaptive discretization of the automatic analytical control of technology products of processing and metallurgical industries it is necessary to automatically measure the current state of the controlled object.

- The predicted behavior of the controlled dynamic object in general case will be different from its actual movement.

- For working in real time a optimization task should be resolved rather quickly, within the allowable delay. 


\section{References}

1. Zolotov Yu A. Fundamental principles of chemistry. In 2 volumes. Vol. 1. General Issues. Separation Procedure: Textbook for higher educational establishments. Moscow: High School; 1999.

2. Sagradyan AL, Suvorovskaya NA. Monitoring of the technological process of flotation Mills. Moscow: Nedra. 1964.

3. Bregman II, Khmara VV, Golant Yu A, Nefed'ev Yu I, Ogol AF. Automated System of Analytical Control (ASAC) at metallurgical plants of non-ferrous metallurgical sector. Moscow: Tsvetmetninformatsia. 1986.

4. Lobotskiy Yu G, Khmara VV. Issues of automatic sampling and transporting the samples of processing and metallurgical production for analysis. Sustainable Development of Mining Regions, International Scientific Journal. 2013; 4:44-9.

5. Itskovich EL. Determining the required frequency of measurements under discrete control. Automation and Telemechanics. 1961; 2:216-23.

6. Kasatkin AS, Kuzmin IV. Assessing the efficiency of automated control systems. Moscow: Energiya; 1967.

7. Itskovich EL. Controlling production with the help of computers. Moscow: Energiya; 1975.

8. Pismenny VV. Determining the frequency of changes un- der discrete control of quality indicators. For Technical Progress, 1974; 10.

9. Stratonovich RL. Information Theory. Moscow: Sovetskoye Radio; 1975.

10. Ovcharenko YeYa. Systematic-information approach to materials' testing when creating AMS. Non-ferrous Metals, 1976; 1:73-7.

11. Nefed'ev Yu I, Khmara VV, Ovcharenko YeYa. ASAC during systematic transition to automated management systems. Automation of the Substance Chemical Analysis. Moscow, Nauka; 1980.

12. Rulnova AZ, Kerentseva LG, Gudima VI, Khmara VV, Bregman II, Akishin NV. Economic efficiency of introducing automated systems and tools of analytical control at the non-ferrous metallurgical plants. Moscow: Tsvetmetinformatsia. 1987.

13. Bishop CM. Neural networks for pattern recognition. Oxford University Press; 1995.

14. Tashatov N. Discretization and restoration of signals. Available from: http://enu.kz/repository/

15. Khmara VV. Optimization of the sampling interval under the discrete control of changing parameter. Non-ferrous metals: Moscow. 2009; 2:97-9.

16. Rannev GG. Information-measurement equipment and electronics. Textbook. Moscow: Academia; 2006. 